\title{
Clinical evaluation of the efficacy and safety of combined bipolar radiofrequency and optical energies vs. optical energy alone for the treatment of pigmented lesions and photo-damage in aging hands
}

\author{
Ines Verner* \\ Dermatologist, Verner Clinic, Tel Aviv, Israel
}

\begin{abstract}
Background: Different treatment modalities are used for the treatment and aesthetic improvement of aging hands. This study evaluated the efficacy and safety of a novel technology, which combines bipolar radiofrequency (RF) and optical energies for the cosmetic treatment of aging hands.

Objective: To assess the efficacy, safety, tolerability and patient satisfaction of combined bipolar radiofrequency and optical energies versus optical energy alone for the treatment of aging hands.

Methods: Thirteen female patients with solar lentigines on the back of the hands were enrolled. Participants received 3 treatments: Combined RF and Intense Pulsed Light (IPL) on one hand and IPL treatment alone on the other. Standardized clinical photographs were taken and patient and investigator improvement assessment (Global Aesthetic Improvement (GAI) scale), patient satisfaction and tolerability were evaluated.

Results: At the 1 and 3 months follow-up skin laxity and pigmentation, investigator and patient improvement assessments and satisfaction, were significantly better in the hand treated with combined bipolar RF and IPL.

Conclusion: This study demonstrates the safety and efficacy of combining RF and optical energies for the aesthetic improvement of aging hands. Combined RF and IPL treatment was more efficient than IPL alone in improving skin pigmentation, skin laxity and texture.
\end{abstract}

\section{Introduction}

Noninvasive skin rejuvenation therapy is becoming increasingly popular in aesthetic medicine, with innovative treatment modalities now also targeting the aging hands, a cosmetic indication that reaches beyond the more traditional aging face and décolletage indications. With noninvasive facial rejuvenation becoming very efficacious in recent years, a patient's true age is becoming apparent on the hands leading to the demand and need for efficacious treatment modalities for this area. Continued research has led to the steady development of ablative and nonablative energy-based devices that are being increasingly used for the cosmetic improvement of aging hands, which can be typically characterized by the development of dyschromias, solar lentigines, solar keratoses, fine telangiectasias, as well as a progressive loss of skin texture and skin atrophy resulting in varying degrees of wrinkling, fine lines and skin laxity that occur as a result of both intrinsic and extrinsic aging processes [1,2]. Topical agents, such as hydroquinone-based preparations and others, have long been employed to lighten and improve the cosmesis of pigmented lesions seen in aging skin; however, these topically applied preparations are not sufficiently effective in the treatment of solar lentigines (a very common complaint) and, moreover, they do not address other aspects photoaging, such as wrinkles, fine lines and skin laxity.

A number of minimally invasive and noninvasive techniques are currently available for the treatment and cosmetic improvement of photo-aged skin ranging from the injection of neurotoxins and a multitude of filler products of varying compositions, chemical peels, to the use of energy-based modalities, such as focused ultrasound, RF, laser and light-based technologies. However, many of the modalities, particularly peels or ablative devices that are currently used to improve the typical signs of photoaging are limited by unwanted side effects that include post-inflammatory hyperpigmentation (PIH), prolonged downtime, low treatment tolerability and a risk for scarring [1-4]. Moreover rejuvenation of the hands has been associated with a higher risk for side effects than facial rejuvenation. Hand skin is thin and has few pilosebaceous units leading to a slow wound healing process and a higher risk for scarring. Thus, only low peel agent concentrations and low energies of energy-based devices can be used in this area.

Ever since their inception, RF-based devices have become very popular in skin rejuvenation therapy due to the excellent treatment outcomes achievable coupled with minimal downtime, a favorable

Correspondence to: Ines Verner, Dr. Verner Clinic Tel Aviv, 39 Jerusalem St., POB 39, Kiriat Ono 55423, Israel, Tel: 972-3-6353183; Fax: 972-3-5341381; E-mail: ines.verner@gmail.com

Key words: bipolar radiofrequency, intense pulsed light, laser, photodamage, pigmentary disorders, noninvasive skin rejuvenation

Received: May 05, 2016; Accepted: May 30, 2016; Published: June 01, 2016 
Verner I (2016) Clinical evaluation of the efficacy and safety of combined bipolar radiofrequency and optical energies vs. optical energy alone for the treatment of pigmented lesions and photo-damage in aging hands

safety profile and a high tolerability associated with the noninvasive treatment approach $[2,5]$. Moreover, the use of RF in skin rejuvenation procedures is proven to be safe and effective in those individuals of darker phototypes due to the nature of the delivery of heat energy to the skin [5]. The use of optical energy in the form of IPL, a broadband light source, has long been viewed as an effective and safe modality for the treatment of solar lentigines, skin rejuvenation and the treatment of photo-damaged skin $[6,8]$; however, the treatment modality has been shown to be only marginally effective for wrinkles and lines. Moreover, frequently the light (IPL) energy needed to achieve clinical results in skin laxity or textural improvement is higher than the skin can tolerate, leading to the risk of side effects such as a prolonged and severe wound healing process, postinflammatory hyper and hypopigmentation and even scarring.

Elōs technology, which combines optical energies (laser or light) with bipolar RF energy, has been proven in previous clinical studies to be a safe and effective treatment modality for the cosmetic improvement of aging and photo-aged skin, while keeping unwanted side effects and downtime to a minimum $[9,10]$. By adding RF energy to light (IPL) energy, the light energy level can be lowered while compensating for this lower light energy by RF energy. In this way there is sufficient energy for the treatment to be effective, while the risk for side effects is kept to a minimum. This compensation for the lowering of light energy by $\mathrm{RF}$ energy not only lowers the risk for side effects, but also allows us to treat darker skin types due to the fact that there is no preference of the $\mathrm{RF}$ energy for absorption in melanin as opposed to light energy (IPL). Moreover, it has been shown in recent years that RF energy is capable of inducing volumetric heating in the dermis leading to new collagen formation, tissue tightening and textural improvement of wrinkles and lines. We assumed that combining these two energies together for skin rejuvenation will not only lead to a better clinical improvement of solar lentigines with a very low risk for side effects, but will also allow for remarkable tissue tightening and textural improvement of the skin surface and of wrinkles. We chose a high risk area such as the hands for this study to be able to effectively demonstrate the efficacy and safety of combining IPL and RF energy.

The purpose of this clinical study was to evaluate and compare the efficacy and safety of a combined RF and IPL energy treatment to IPL energy only treatment for the improvement of aging and photodamaged skin on the back of the hands.

\section{Device description}

All subjects were treated with the elōs Plus system (Syneron Medical, Yokneam, Israel). Optical energy (either laser or light) is emitted and preheats dermal structures, which then creates a temperature differential between the targeted structures and the surrounding tissues. Movement of charged particles between the $\mathrm{RF}$ electrodes, through preheated dermal structures where there is lower impedance, generates further heating of the target structures. Addition of RF energy allows for lower optical energy to be used than in traditional light-based systems. The utilization of lower energy levels potentially allows for much safer treatments, even in darker Fitzpatrick Skin Types, resulting in minimal downtime and an excellent safety profile. The system incorporates an active dermal monitoring feature to monitor changes in skin impedance during pulse delivery, so that optimal energy is delivered to target structures, while protecting the surrounding tissues.

In this study, we utilized the SRA Plus (Skin Rejuvenation Advanced) applicator for the treatment of the typical signs of skin aging. The applicator delivers pulsed light optical energy at 470-980 nm wavelengths, and bipolar RF energy, which has been found in previous clinical trials to be ideal for photorejuvenation treatments. The hand piece delivers light fluence of up to $45 \mathrm{~J} / \mathrm{cm}^{2}$ and RF energy up to $25 \mathrm{~J} /$ $\mathrm{cm}^{2}$, and covers a treatment area of $25 \times 12 \mathrm{~mm}$.

\section{Study design}

This was a prospective, open label, single center clinical trial that included 13 healthy Caucasian female patients, ranging in age from 47 to 75 years (mean age 64 years) with Fitzpatrick Skin Type III, who presented with pigmented lesions and other hallmark signs of skin aging on the backs of the hands. Using the SRA Plus applicator, all study participants received a total of three treatment sessions spaced 3 to 4 weeks apart. Randomly one hand received IPL and RF energy and the other hand received IPL alone. Treatment parameters increased slightly on average from the $1^{\text {st }}$ to the $2^{\text {nd }}$ treatment with $14.2 \mathrm{~J} / \mathrm{cm}^{3}$ of RF increasing to $17 \mathrm{~J} / \mathrm{cm}^{3}$ on the hand treated with combined energies and $9.7 \mathrm{~J} / \mathrm{cm}^{2}$ of IPL energy increasing to $10.2 \mathrm{~J} / \mathrm{cm}^{2}$ for the $2^{\text {nd }}$ and $3^{\text {rd }}$ treatments. The optical energy used was identical in both study arms.

Patients were followed-up at baseline and at 1 and 3 months after the final treatment session. Clinical photographs were taken in a standardized manner using the Profect ${ }^{\oplus}$ Photography System (Profect ${ }^{\mathrm{m}}$ Medical Technologies, Vancouver, BC, Canada) and the Antera 3D (Miravex Ltd., Dublin, Ireland) for 3D photography at baseline and at each follow-up visit, and comparisons were made at the end of the clinical trial. A number of study evaluations were performed and recorded, including patient and investigator improvement assessments made at each follow-up time point using the Global Aesthetic Improvement (GAI) scale (0-4) with ' 0 '=worsened and ' 4 '=marked improvement. Other evaluations included patient satisfaction using a 0-4 point scale ( $0=$ unsatisfied; $4=$ significantly satisfied), and tolerability of treatment using a $0-10$ point scale $(0=$ no discomfort at all; $10=$ intolerable pain). The safety of treatments was assessed by the frequency and severity of the adverse events reported throughout the clinical trial. All study participants met the inclusion/exclusion criteria, and written informed consent was obtained from all patients prior to the initiation of the clinical trial.

\section{Results}

All patients tolerated the treatments without the need for any topical anaesthesia and completed successfully the clinical trial. None had experienced any adverse events. The average physician assessment values at 1 -month and 3-month post-treatment in the combined RF and IPL treated hands were 3.7 and 3.8, respectively, compared to 2.1 and 2.5 for the IPL-energy treated hands (Figure 1). Subject assessments were very similar to the physician assessments and also showed increased improvement in the combined treated hands at both followups. All patients had a faster and more pronounced improvement in solar lentigines on the IPL and RF-treated side with remarkable results already after the 2nd treatment. A typical example is shown in Figure 2. Textural improvement was also much more remarkable on the IPL and RF-treated hand.

Patient satisfaction from treatment and clinical outcomes were also reported to be improved in the RF and IPL treated hands compared to the IPL-energy treated hands with $71 \%$ of subjects reporting that they were significantly satisfied with outcome on the combined treated hand (Figure 3).

As expected, the study participants associated the combined RF 
Verner I (2016) Clinical evaluation of the efficacy and safety of combined bipolar radiofrequency and optical energies vs. optical energy alone for the treatment of pigmented lesions and photo-damage in aging hands

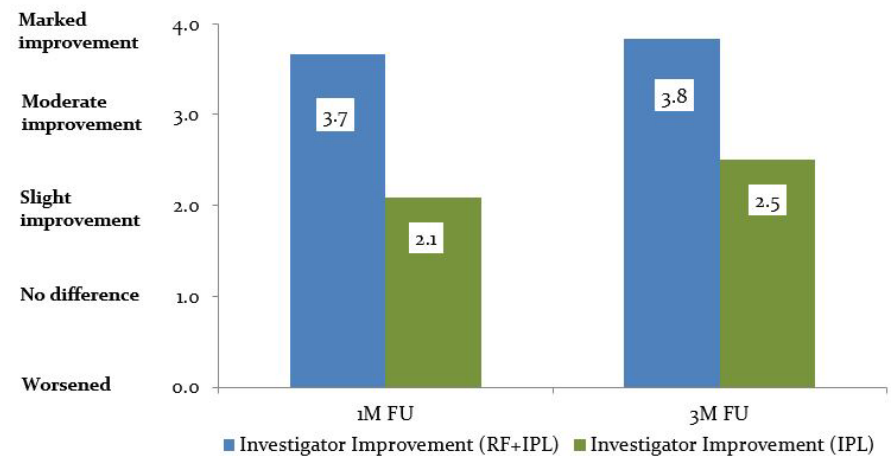

Figure 1. Physician assessment of improvement for both treatment modalities at the 1 and 3-month follow-up visits.

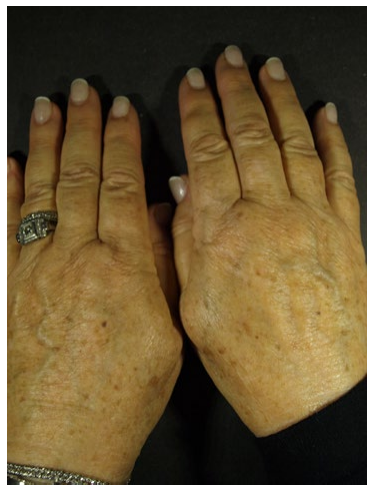

Figure 2a. 71-year-old female with pigmentation and photo-aged hands before treatment.

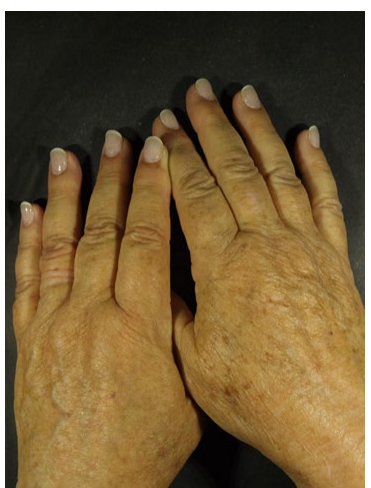

Figure 2b. Same patient's hands after 3 combined RF and IPL treatments to the right hand and 3 IPL-only treatments to the left hand.

and IPL treatment with more pain (moderate on average) compared to the IPL-only treated hand (mild on average). A mild, but transient, erythema was seen in both treated hands with a higher incidence observed in the hands treated with the combined energies ( $92 \% v s$. $38 \%)$. In all of the physician and patient assessments, data showed that improved results were achieved with the combined RF and IPL treatment when compared to the IPL energy only treatment.

\section{Discussion}

The processes of both intrinsic and extrinsic aging contribute to a number of aesthetic changes seen in aging skin including the development of dyschromias, keratoses, solar lentigines, fine telangiectasias, a progressive loss of skin texture with, wrinkles, fine lines and sagging skin. Several treatment modalities are currently being

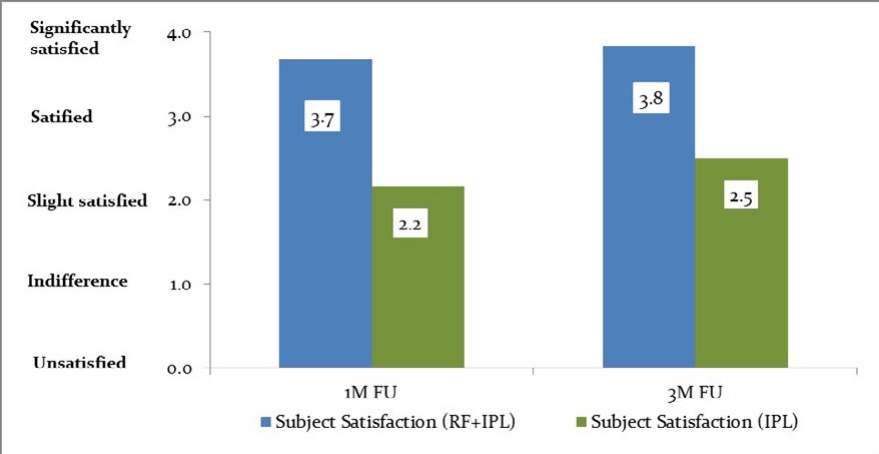

Figure3. Patient satisfaction of treatment for both treatment modalities at the 1 and 3 month follow-up visits.

used to address some of different aspects of the aging skin including peels, ablative and non-ablative lasers, focused ultrasound, RF-based devices as well as combined RF and optical energy modalities with each modality demonstrating varying degrees of success, safety and tolerability $[1,3,11-17]$.

The use of these different modalities and approaches in limited by patient tolerability, wound healing response and time and the risk for side effects such as postinflammatory hyper and hypopigmentation and scarring. Moreover we see nowadays a higher demand for rejuvenation of skin areas off the face, such as the neck, décolleté and the hands. These areas have fewer pilosebaceous units, a slower wound healing process and a higher risk for complications.

IPL has been shown in previous studies to be useful in the treatment of solar lentigines and general skin rejuvenation with improvement of the cosmesis of the aging skin [6-8]; however, the broadband light source works largely superficially and, therefore, is less effective for wrinkles, lines and skin tightening. RF energy, on the other hand, has been shown to be very effective in the treatment of wrinkles and lines and other aspects of the aging skin $[2,4,5,9,10,12,18-20]$. In this study, we compared the efficacy, safety and tolerability of a combined RF and IPL treatment to IPL energy only treatment in the aging hands of 13 healthy Caucasian female patients aged 47 to 75 years (mean age 64 years) with Fitzpatrick Skin Type III. At each of the follow-up visits, results showed that the combined RF and IPL energy treatment was superior to the IPL energy only treatment for the improvement of aging and photoaged skin on the back of the hands, reflected in the higher physician and patient GAI scores. The RF and IPL-treated hands not only showed improved lightening and clearing of pigmented lesions (mainly solar lentigines), but also achieved a much smoother skin when compared to the IPL energy only treated hands. The appreciable improvement in the skin's smoothness is more likely due to neocollagenesis and elastogenesis processes that take place in the targeted skin following the application of the RF energy, which targets the dermis volumetrically, and less likely from the impact of the applied IPL energy, which is more superficial in the skin. The synergistic effects of the RF and IPL energies lead to the superior clinical outcomes seen with this treatment approach compared to the IPL energy only treatment approach.

Patient satisfaction was also found to be significantly higher for the RF and IPL treated hands compared to the IPL energy only treated hands, correlating with the clinically visible results. Interestingly, all patients but one wanted the IPL-only side to be retreated by the combined IPL and RF modality after completing the study. Patients did, however, associate the combined treatment with somewhat more discomfort (moderate on average) compared to the IPL energy only 
Verner I (2016) Clinical evaluation of the efficacy and safety of combined bipolar radiofrequency and optical energies vs. optical energy alone for the treatment of pigmented lesions and photo-damage in aging hands

treatment (mild on average), which was expected due to the increased heat energy generated by the applied RF energy in the targeted skin. A mild but transient post treatment erythema was seen in both study arms and as expected, a higher percentage of patients observed the erythema following the combined treatment of the hands compared to the IPL-only treated hands.

The combination of both the RF and IPL energies, as opposed to IPL treatment alone, resulted in a synergistic effect of the applied energies in the targeted dermis, stimulating most probably collagen remodeling, neocollagenesis and elastogenesis. The combined treatment approach not only led to lightening and clearance of pigmented lesions, but also to a visually appreciable improvement in the smoothness and laxity of the skin with minimal procedural downtime, resulting in a more complete skin rejuvenation of the back of the hands.

\section{Conclusion}

The clinical trial data presented here robustly support the efficacy and safety of a combined RF and IPL treatment approach compared to IPL only when treating aging hand skin. The elōs Plus device using the SRA Plus applicator appears to be an excellent treatment modality for the aesthetic improvement of benign pigmented lesions, as well as for overall skin rejuvenation of the aging hands, particularly when $\mathrm{RF}$ and IPL energies are combined, due to the minimal downtime and minimal risk coupled with the superior clinical outcomes associated with treatment.

\section{References}

1. Lolis MS, Goldberg DJ (2012) Radiofrequency in cosmetic dermatology: a review. Dermatol Surg 38: 1765-1776. [Crossref]

2. Bloom BS, Emer J, Goldberg DJ (2012) Assessment of safety and efficacy of a bipolar fractionated radiofrequency device in the treatment of photodamaged skin. $J$ Cosmet Laser Ther 14: 208-211.[Crossref]

3. Alexiades-Armenakas MR, Dover JS, Arndt KA (2008) The spectrum of laser skin resurfacing: nonablative, fractional, and ablative laser resurfacing. J Am Acad Dermatol 58: 719-737. [Crossref]

4. Hammes S, Greve B, Raulin C (2006) Electro-optical synergy (ELOS) technology for nonablative skin rejuvenation: a preliminary prospective study. J Eur Acad Dermatol Venereol 20: 1070-1075. [Crossref]

5. Man J, Goldberg DJ (2012) Safety and efficacy of fractional bipolar radiofrequency treatment in Fitzpatrick skin types V-VI. J Cosmet Laser Ther 14:179-183. [Crossref]
6. Weiss RA, Weiss MA, Beasley KL (2002) Rejuvenation of photoaged skin: 5 years results with intense pulsed light of the face, neck, and chest. Dermatol Surg 28: 11151119. [Crossref]

7. Sadick NS (2003) Update on non-ablative light therapy for rejuvenation: a review. Lasers Surg Med 32: 120-128. [Crossref]

8. Bitter PH (2000) Noninvasive rejuvenation of photodamaged skin using serial, fullface intense pulsed light treatments. Dermatol Surg 26: 835-843. [Crossref]

9. Sadick NS1 (2005) Combination radiofrequency and light energies: electro-optica synergy technology in esthetic medicine. Dermatol Surg 31: 1211-1217. [Crossref]

10. El-Domyati M, El-Ammawi TS, Medhat W, Moawad O, Mahoney MG, et al. (2010) Electro-optical synergy technique. A new and effective nonablative approach to skin aging. J Clin Aesthet Dermatol 3: 22-30. [Crossref]

11. Hantash BM, Gladstone HB (2009) Current role of resurfacing lasers. G Ital Dermatol Venereol 144: 229-241. [Crossref]

12. Brightman L, Goldman MP, Taub AF (2009) Sublative rejuvenation: experience with a new fractional radiofrequency system for skin rejuvenation and repair. $J$ Drugs Dermatol 8: s9-13. [Crossref]

13. Atiyeh BS, Dibo SA (2009) Nonsurgical nonablative treatment of aging skin: radiofrequency technologies between aggressive marketing and evidence-based efficacy. Aesthetic Plast Surg 33: 283-294. [Crossref]

14. Bogle MA, Dover JS (2009) Tissue tightening technologies. Dermatol Clin 27: 491499. [Crossref]

15. Berube D, Renton B, Hantash BM (2009) A predictive model of minimally invasive bipolar fractional radiofrequency skin treatment. Lasers Surg Med 41: 473-478. [Crossref]

16. Chan HH (2005) Effective and safe use of lasers, light sources, and radiofrequency devices in the clinical management of Asian patients with selected dermatoses. Lasers Surg Med 37: 179-185. [Crossref]

17. Collawn SS (2010) Skin tightening with fractional lasers, radiofrequency, Smartlipo. Ann Plast Surg 64: 526-529. [Crossref]

18. Doshi SN, Alster TS (2005) Combination radiofrequency and diode laser for treatment of facial rhytides and skin laxity. $J$ Cosmet Laser Ther 7: 11-15. [Crossref]

19. Sadick NS, Alexiades-Armenakas M, Bitter P Jr, Hruza G, Mulholland RS (2005) Enhanced full-face skin rejuvenation using synchronous intense pulsed optical and conducted bipolar radiofrequency energy (ELOS): introducing selective radiophotothermolysis. J Drugs Dermatol 4: 181-186. [Crossref]

20. Alexiades-Armenakas M (2006) Rhytides, laxity, and photoaging treated with a combination of radiofrequency, diode laser, and pulsed light and assessed with a comprehensive grading scale. J Drugs Dermatol 5: 731-738. [Crossref]

Copyright: (C2016 Verner I. This is an open-access article distributed under the terms of the Creative Commons Attribution License, which permits unrestricted use, distribution, and reproduction in any medium, provided the original author and source are credited. 